\title{
Electronically beamscannable sinusoidally modulated reactance surface antenna
}

\author{
Doohyun Yang* and Sangwook Nam \\ Institute of New Media and Communications, Department of Electrical Engineering, Seoul National University, Seoul, \\ South Korea
}

Received: 26 September 2018 / Accepted: 3 February 2019

\begin{abstract}
An electronically beamscannable sinusoidally modulated reactance surface (SMRS) antenna and its design procedure are investigated. The antenna is composed of capacitively modulated reactance surface whose profile is a sinusoidally varying form. This configuration generates a radiating leaky wave and the antenna's radiation pattern including beam angle and beamwidth can be controlled with different parameters of the modulated surface reactance of the SMRS period. A beamscanning characteristic of the capacitively modulated SMRS antenna is shown with the design procedure and the simulated results. Designed antenna was simulated using commercial EM tool and the result was well matched with the calculated main beam direction verifying the validity of design method. About $33^{\circ}$ of beamcanning range was obtained with the center radiating angle of $45^{\circ}$ at $9 \mathrm{GHz}$. Designed antenna showed reasonable input matching and efficiencies within beamscanning range of the antenna.
\end{abstract}

Keywords: Metasurfaces / leaky wave antennas / sinusoidally modulated reactance surfaces / beamscanning antennas / reconfigurable antennas

\section{Introduction}

The initial analysis and investigation on the guidance and radiation properties of sinusoidally modulated reactance surfaces (SMRS) were done by Oliner and Hessel in 1959 [1]. After this first research on the SMRS, there have been some researches utilizing this kind of concept for the application to the antennas [2-8]. Due to the periodic structure of the modulated reactance surface, this SMRS antenna is categorized as a periodic leaky wave antenna [9]. The SMRS antenna implemented on the PCB was introduced and its design procedure and its radiating wave characteristics were investigated [3]. The control of the surface reactance, which is defined as the ratio of the tangential electric field to the tangential magnetic field, was achieved by varying the gaps between the conductor strips on top of the grounded dielectric substrate of the unit cell. The SMRS antenna has attracted a great deal of interests due to their low profile, light weight, and highly directive beam pattern characteristics and engineered for lots of applications [4-8]. However, in spite of these advantages of SMRS antennas, beamscanning property which is the inherent characteristic of leaky wave antennas

\footnotetext{
* e-mail: doohuyn@ael.snu.ac.kr
}

$[9,10]$ limits the application of the SMRS antenna to the areas which require linkage between transmitter and receiver on moving platforms at a fixed frequency.

After few years from the initial application of the SMRS to the antenna, new concept for the implementation of the SMRS with the different configuration was proposed [4]. This work achieved the sinusoidal modulation of the surface reactance by varying the loading capacitances between the conductor strips instead of variation of the gaps between the conductors. Also a new method for the calculation of the surface reactances using driven mode instead of eigenmode in EM simulation tool was suggested.

To circumvent the beamscanning property of the SMRS antenna, there were some researches implementing SMRS antenna on the reconfigurable material such as graphene and liquid crystal $[11,12]$ for the radiation beam angle control of the antenna at a fixed frequency. Both of these researches provide electronical reconfigurability for the radiation pattern of the antenna by controlling the conductance of the graphene and the direction of optical axis from the value of bias voltages, respectively.

In this paper, we propose an SMRS antenna whose surface reactance is controlled by the capacitances between the conductor strips for the radiation pattern reconfigurability at a fixed frequency. Different sets of the capacitances give the different values of radiation beam 


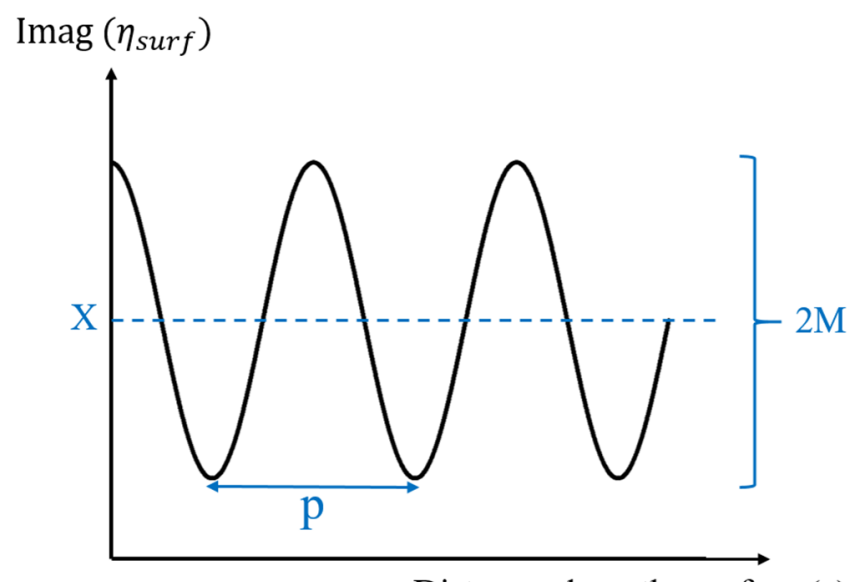

Distance along the surface (z)

Fig. 1. Plot of the surface reactance of the SMRS.

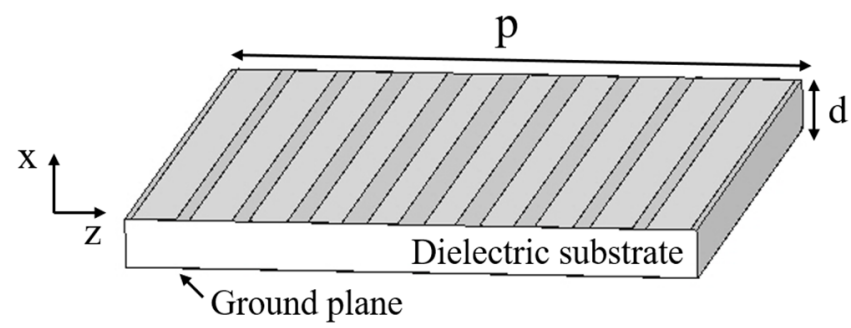

Fig. 2. A unit period of SMRS antenna composed of 10 metallic strips on a dielectric substrate over ground plane.

angle. Detailed design procedure will be introduced and results were simulated using commercial EM tool [Computer Simulation Technology (CST)]. The controllability of the radiating beam angle according to the calculated capacitance sets implies that the antenna can be implemented as a reconfigurable beamscanning antenna with the components such as varactor diodes.

\section{Surface reactance calculation of the capacitively modulated SMRS}

\subsection{Theoretical concept}

In this section, we are going to briefly introduce the basic theory of SMRS antenna from the referenced work $[1,3]$. Detailed theories and the basic principles of SMRS antenna are well organized in [1] and [3].

An SMRS has a sinusoidally varying surface reactance where the surface reactance refers the ratio of the tangential component of the magnetic field to the tangential component of the electric field. The reactance of the SMRS can be expressed in mathematical form with the equation below

$$
\eta_{\text {surf }}(z)=j \eta_{0} X^{\prime}\left[1+M \cos \left(\frac{2 \pi z}{p}\right)\right]
$$

where $X$ is the average surface reactance, $M$ is the modulation factor, $p$ is the period of the sinusoidal function, and $X^{\prime}$ is the average surface reactance divided

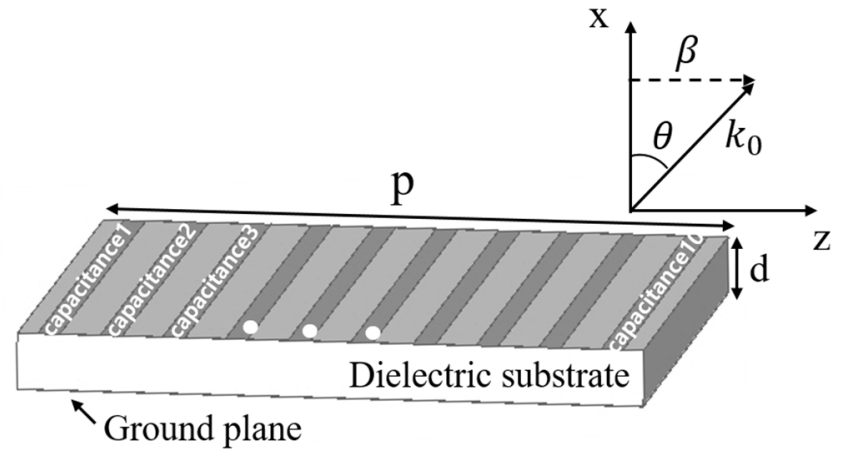

Fig. 3. A unit period of SMRS antenna composed of capacitively modulated reactance surface and its wavenumber notations.

by the free-space wave impedance $\left(X^{\prime}=X / \eta_{0}\right)$. This form of the surface reactance can be plotted as Figure 1. As a primitive configuration of this SMRS, an array of conductor strips over the ground plane with different gap sizes between them is widely used as shown in Figure 2 .

When the unit period of Figure 2 is placed periodically, application of the SMRS as an antenna is available due to the Floquet modes from the periodicity of the SMRS. Most of SMRS antenna utilizes -1st Floquet mode as a main radiating mode. With the appropriate determination of the surface reactance modulation parameters in (1), some of guided wave modes on the SMRS become leaky wave modes and can be utilized as a leaky wave antenna. Based on the equation (18) of [1], the perturbated solution of wavenumber of guided wave assuming the small modulation amplitude $(M<0.6)$ leads to a conclusion that the variables $X$ and $p$ of equation (1) control phase constant $(\beta)$ which contributes to the beam angle of the radiating wave and $M$ controls the attenuation constant $(\alpha)$ which leads to the determination of the beamwidth of the antenna.

\subsection{SMRS antenna composed of capacitively modulated reactance surface}

Based on the theoretical concept and feasibility of implementation of the SMRS with the varying gaps between conductor strips as shown in Figure 2, a recent work proposed a new configuration for the SMRS composed of equal gaps between conductor strips and different loading capacitances between the strips to achieve a sinusoidally varying surface reactances [4] like unit period model in Figure 3. Inspired from the referenced work, we investigated the beamscanning characteristic of the SMRS antenna composed of capacitively modulated reactance surface according to the pre-determined capacitance sets at a fixed frequency.

To design the SMRS leaky wave antenna with the desired radiation angle at a fixed frequency, phase constant of -1st Floquet mode should be calculated using from the perturbed solution of the dispersion of the SMRS [1]

$$
\beta_{-1} \approx \beta_{\text {approx }}-\frac{2 \pi}{p}=k_{0} \sqrt{1+X^{\prime 2}}-\frac{2 \pi}{p}
$$

where $\beta_{\text {approx }}$ is approximated phase constant of fundamental guided mode when the $M=0$ is assumed. With this 


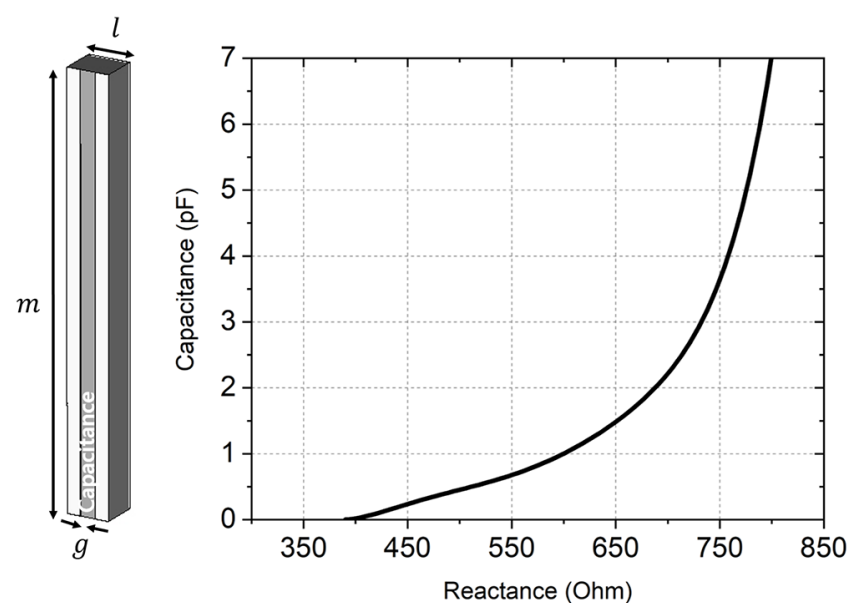

Fig. 4. A unit cell structure and the plot of surface reactance against loading capacitance.

calculated phase constant of -1 st Floquet mode, radiation angle of the SMRS antenna can be calculated from the wavenumber notation of Figure 3.

$$
\sin \left(\theta_{n=-1}\right)=\frac{\beta_{-1}-\frac{2 \pi}{p}}{k_{0}} .
$$

In addition to the -1 st Floquet mode, -2 nd Floquet mode radiation angle also can be obtained putting -2 nd Floquet mode harmonic phase instead of -1 st harmonics phase in the above equation. For the calculation of the desired radiation angle of the SMRS antenna, calculation of the surface reactance according to the loading capacitance at a given structure is necessary. In Figure 4, we have calculated the surface reactance of the unit cell against the loading capacitance between the conductor strips using eigenmode solver. The unit cell model is designed on the Rogers RT 6006 substrate whose dielectric constant is 6.15 with the thickness of $2.54 \mathrm{~mm}$ and its surface reactance at the $9 \mathrm{GHz}$ can be calculated applying the transverse resonance condition to the equivalent transmission line model of the unit cell

$$
\eta_{\text {surf }}=j \eta_{0} \sqrt{\left(\frac{\phi}{k_{0} l}\right)^{2}-1}
$$

where $\phi$ is the phase difference across the unit cell at $9 \mathrm{GHz}$ obtained from the eigenmode solver simulation and $l$ is the length of the unit cell as shown in the geometry of Figure 4. The detailed dimensions of our simulated unit cells are as follows. $m=28.25 \mathrm{~mm}, l=2.825 \mathrm{~mm}$, and $g=1 \mathrm{~mm}$. The variation of the loading capacitance assumes the situation for the implementation of the antenna using varactor diodes which provide different loading capacitance values between the conductor strips in the unit cell according to the bias DC voltages. With the calculated surface reactance of the unit cell versus the values of the loading capacitances, we can control the radiating angle of the SMRS leaky wave antenna and the details are going to be introduced in the next section.

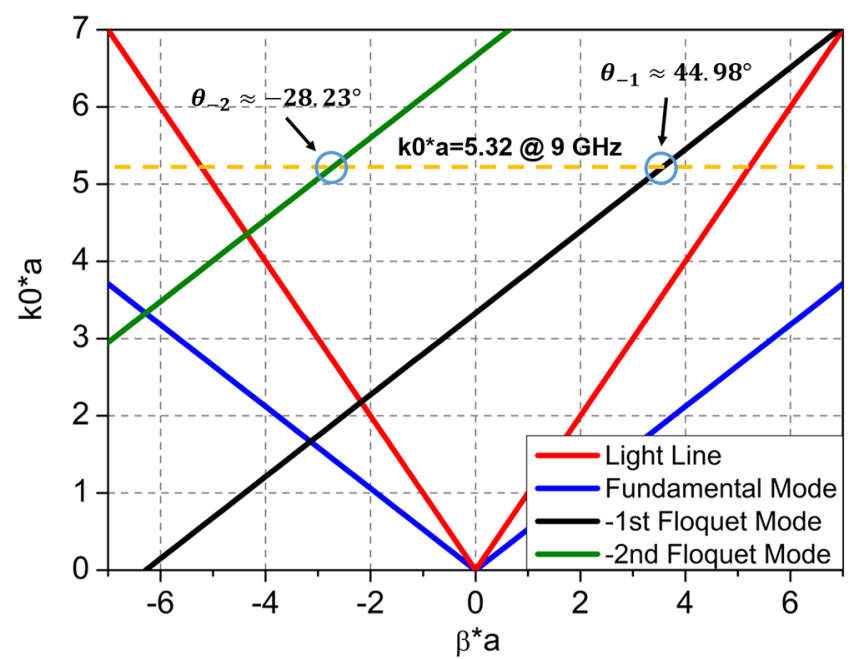

Fig. 5. Dispersion diagram of the proposed antenna based on (1) when $X^{\prime}$ is set to 1.6 .

\section{Electronically beamscannable SMRS antenna design}

The radiation angle of the SMRS antenna calculated from (3) at a fixed frequency is dependent on the average value of normalized surface reactance $X^{\prime}$ and the period of unit cell $p$ as referred in previous section. If we determine the unit period of the SMRS antenna for a certain period value $p$, the radiation angle of the SMRS antenna can be controlled within the range of the achievable surface reactance of the unit cell. For our case, the period of the SMRS antenna is chosen as $28.25 \mathrm{~mm}$.

Figure 5 shows the dispersion relation of the structure composed of unit periods of Figure 3 with the parameters $X^{\prime}=1.6, M=0.15$. This dispersion characteristic of the structure is plotted based on (2) which is an approximated expression of the propagation constant of the guided wave on the SMRS structure.

The dashed orange line stands for the $k 0^{*} a$ value at the operation frequency of $9 \mathrm{GHz}$ so the crossing points of the dispersion curves with this line mean the radiation point of the proposed antenna when the point is within the light line region. The dispersion relation of the guided structure with different parameters can be obtained using similar procedure. The radiation angle of -1 st Floquet mode can be calculated using (2).

In addition to the dispersion relation of the structure, the attenuation constant of the structure based on the equation (18) of [1] is plotted in Figure 6. The attenuation constant does not vary much according to the frequency change within our band. Actually the attenuation constant of the SMRS antenna changes dominantly by the surface reactance modulation amplitude $M$ as introduced in the Section 2.1. So for this SMRS antenna, the amount of radiation is determined from the surface reactance modulation amplitude and the beamwidth of the antenna is determined with this parameter.

From (1) and (3) we have calculated the required values of the average surface reactances of unit period to achieve the pre-determined SMRS antenna radiation angles and 


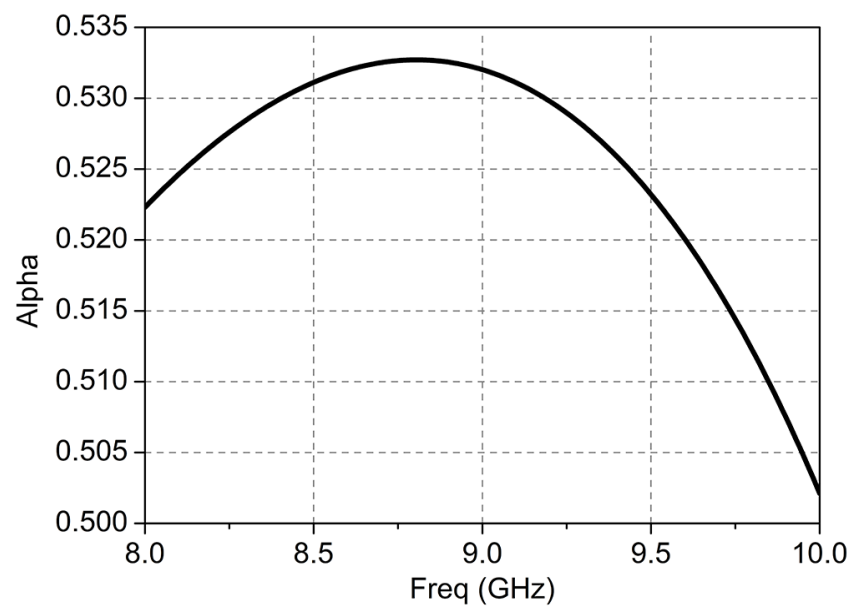

Fig. 6. Attenuation constant of the proposed antenna versus frequency based on the equation (18) of [1] when $X^{\prime}$ is set to 1.6 .
Table 1. Information of surface reactance sets for the different desired radiation angles at $9 \mathrm{GHz}$.

\begin{tabular}{llll}
\hline Set \# & $X^{\prime}$ & $X(\mathrm{Ohm})$ & $\theta_{-1}$ \\
\hline 1 & 1.4 & 527.8 & $32.72^{\circ}$ \\
2 & 1.5 & 565.5 & $38.52^{\circ}$ \\
3 & 1.6 & 603.2 & $44.98^{\circ}$ \\
4 & 1.7 & 640.9 & $52.4^{\circ}$ \\
5 & 1.8 & 678.6 & $61.54^{\circ}$ \\
\hline
\end{tabular}

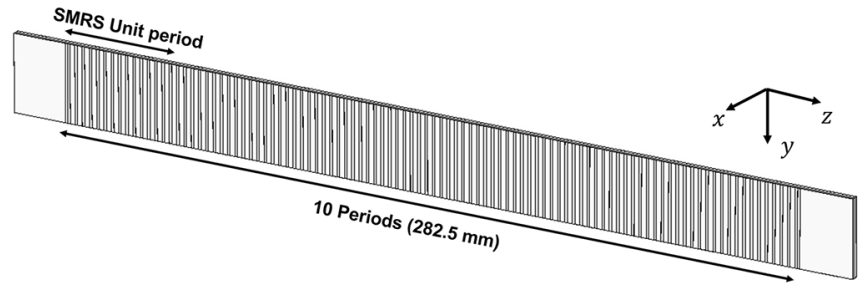

Fig. 7. 3D model of the designed SMRS antenna composed of 10 uniform periods for the EM simulation.

Table 2. Required surface reactances and loading capacitances at each unit cell according to the set number from Table 1.

\begin{tabular}{lllllll}
\hline Set \# & Parameter & Cell 1 & Cell 2 & Cell 3 & Cell 4 & Cell 5 \\
\hline 1 & $X$ (Ohms) & 603.1 & 574.3 & 527.8 & 481.3 & 452.5 \\
& Capacitance $(\mathrm{pF})$ & 1.028 & 0.818 & 0.571 & 0.377 & 0.249 \\
2 & $X$ (Ohms) & 646.2 & 615.4 & 565.5 & 515.6 & 484.8 \\
& Capacitance $(\mathrm{pF})$ & 1.44 & 1.133 & 0.764 & 0.522 & 0.392 \\
3 & $X$ (Ohms) & 689.3 & 656.4 & 603.2 & 550 & 517.2 \\
& Capacitance $(\mathrm{pF})$ & 2.025 & 1.548 & 1.029 & 0.677 & 0.524 \\
4 & $X$ (Ohms) & 732.3 & 697.4 & 640.9 & 584.4 & 549.5 \\
& Capacitance $(\mathrm{pF})$ & 3.003 & 2.169 & 1.383 & 0.886 & 0.675 \\
5 & $X$ (Ohms) & 775.4 & 783.3 & 678.6 & 618.8 & 581.8 \\
& Capacitance $(\mathrm{pF})$ & 4.993 & 3.202 & 1.858 & 1.163 & 0.868 \\
\hline
\end{tabular}

tabulated in Table 1 . The modulation amplitude $M$ is determined to 0.15 which is within the small $M$ range assumed for the perturbed solution of the dispersion of the SMRS. Based on (1) and from the calculated parameters sets of Table 1 with surface reactance range shown in Figure 4, required surface reactance values and their corresponding loading capacitances at each sample point were calculated in Table 2. A period of an SMRS is composed of 10 unit cells and has even symmetry from the midpoint of the unit period so only half of the information of unit period is shown.

\section{Simulated results}

For the verification of our calculation for the beamscannability of the capacitively modulated SMRS antenna, we have designed and simulated the SMRS antenna model. Overall performance of the proposed antenna was simulated using commercial EM tool CST and constructed
3D EM model of the SMRS antenna is shown in Figure 7. The antenna is composed of 10 uniform unit periods with different loading capacitances within each unit cell. The dimensions of the unit period and unit cell are the same as referred in previous sections. Parallel plate feed structures were added at input and output ports for the transition and excitation of the signal.

Gain patterns of the capacitively modulated SMRS antenna according to the surface reactance sets at $9 \mathrm{GHz}$ are shown in Figure 8. Main beam directions of the each pattern are quiet well matched with calculated predictions in Section 3. The calculated beam angles and simulated results are compared in Table 3 . About $33^{\circ}$ of the beamscanning range from $32^{\circ}$ to the $65^{\circ}$ of the main beam was observed at a fixed frequency. Due to the existence of the -2 nd Floquet mode within the fast wave region at the operation frequency, there is small lobe radiating around $-30^{\circ}$ of each pattern. The result of Set 1 shows the highest gain value with $16.6 \mathrm{~dB}$ and gradual gain drop was occurred as the radiation beam angle increases; about $2 \mathrm{~dB}$ of gain 


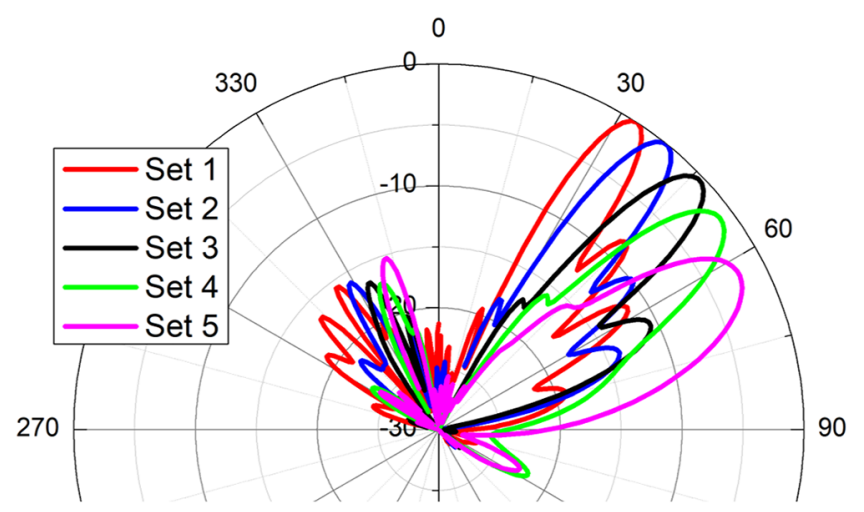

Fig. 8. Gain patterns of the SMRS antenna at $9 \mathrm{GHz}$ according to the caclulated surface reactance sets in Section 3.

Table 3. Comparison of the calculated radiation angles and simulated radiation angles according to the set numbers at $9 \mathrm{GHz}$.

\begin{tabular}{lll}
\hline Set \# & Calculation & Simulation \\
\hline 1 & $32.72^{\circ}$ & $33^{\circ}$ \\
2 & $38.52^{\circ}$ & $39.2^{\circ}$ \\
3 & $44.98^{\circ}$ & $46.3^{\circ}$ \\
4 & $52.4^{\circ}$ & $53.2^{\circ}$ \\
5 & $61.54^{\circ}$ & $63^{\circ}$ \\
\hline
\end{tabular}

drop was observed in Set 5 case when compared to the result of Set 1.

The S-parameter results of the designed SMRS antenna sets are shown in Figures 9 and 10. The input matching (S11) of the SMRS antenna sets is well achieved within the calculated beamscan range at $9 \mathrm{GHz}$ and keep below $-10 \mathrm{~dB}$ level from $8 \mathrm{GHz}$ to $10 \mathrm{GHz}$ frequency band. The transmission coefficient (S21) shows that the majority of the input power of the SMRS antenna sets is radiating at $9 \mathrm{GHz}$. The dips shown in S21 of Sets 4 and 5 are due to the resonance-line behavior of each set as can be seen in input matching results. The radiation efficiencies of the SMRS antenna sets are 78 for Set 1, 79 for Set 2, 80 for Set 3, 80 for Set 4 , and 82 for Set 5 at $9 \mathrm{GHz}$, respectively.

\section{Conclusion}

Beamscanning characteristic of the capacitively modulated SMRS antenna at a fixed frequency was investigated. The unit cell model is composed of two parallel conductor strips and loading capacitance between the strips above the ground plane and allowable surface reactance values according to the loading capacitance were calculated using eigenmode simulation. Different surface reactance sets for the different radiation angles were calculated based on the

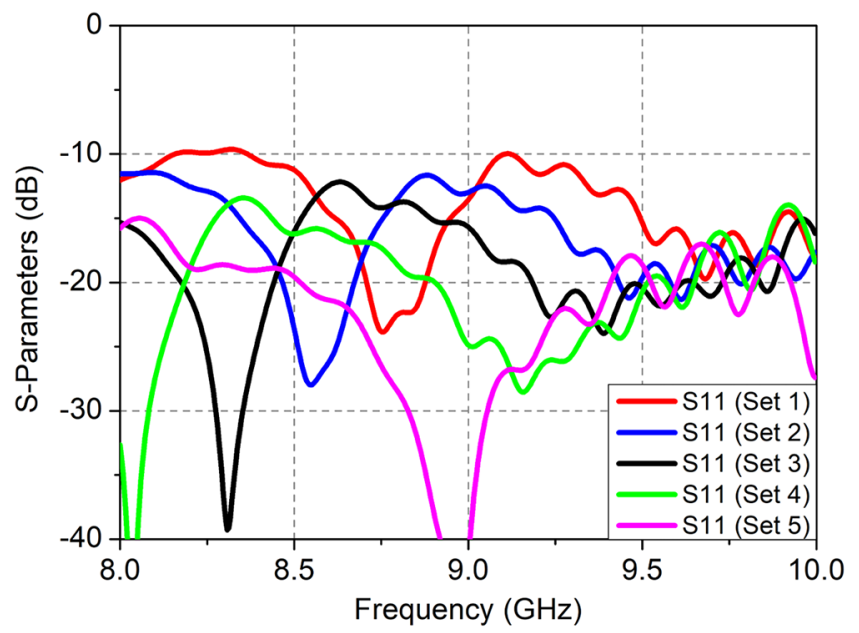

Fig. 9. Simulated result of the S11 of the designed antenna according to the set numbers.

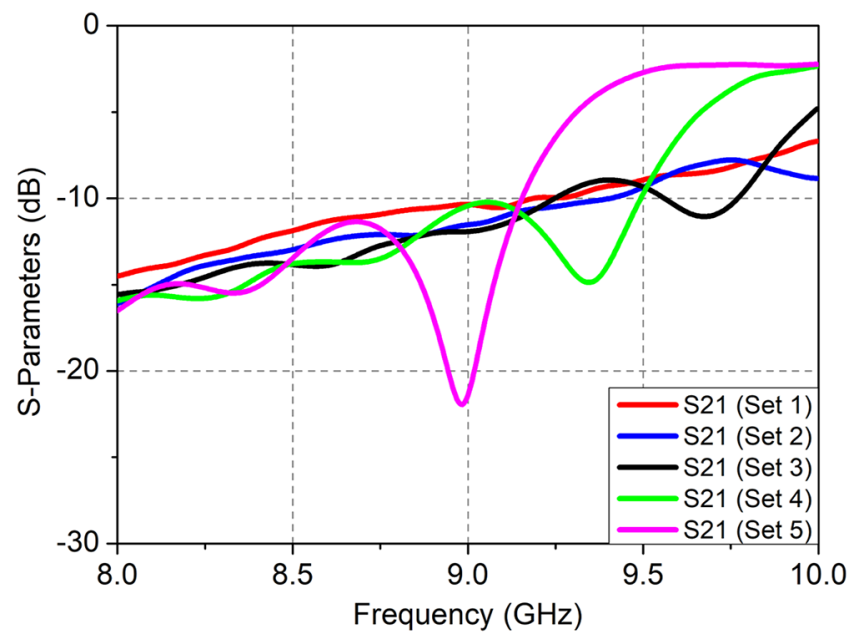

Fig. 10. Simulated result of the $\mathrm{S} 21$ of the designed antenna according to the set numbers.

principle of the SMRS antenna that the radiation angle of the antenna depends on the average value of the modulated surface reactance. The simulated radiation patterns according to the surface reactance sets verify our design procedure of the SMRS antenna and about $33^{\circ}$ of beam scan range was obtained at $9 \mathrm{GHz}$ frequency. Although the beamscanning with the fabricated SMRS antenna model has not achieved in this paper, our work provides the possibility of the electronical beamscanning of the SMRS and verified the feasibility of the idea throughout the paper.

This work was supported by the Center for Advanced Meta-Material (CAMM) funded by the Ministry of Science, ICT, and Future Planning as Global Frontier Project (CAMM2014M3A6B3063708). 


\section{References}

1. A.A. Oliner, A. Hessel, Guided waves on sinusoidallymodulated reactance surfaces, IRE Trans. Antennas Propag. 7, $201(1959)$

2. B. Fong, J. Colburn, J. Ottusch, J. Visher, D. Sievenpiper, Scalar and tensor holographic artificial impedance surfaces, IEEE Trans. Antennas Propag. 58, 3212 (2010)

3. A.M. Patel, A. Grbic, A printed leaky-wave antenna based on a sinusoidally-modulated reactance surface, IEEE Trans. Antennas Propag. 59, 2087 (2011)

4. A.H. Panaretos, D.H. Werner, Leaky-wave antennas based on capacitively tuned modulated reactance surfaces, IEEE Antennas Wirel. Propag. Lett. 15, 678 (2015)

5. C.A. Balanis, M.A. Amiri, A.Y. Modi, S. Pandi, C.R. Birtcher, Applications of AMC-based impedance surfaces, EPJ Appl. Metamater. 5, 3 (2018)

6. G. Minatti, F. Caminita, M. Casaletti, S. Maci, Spiral leakywave antennas based on modulated surface impedance, IEEE Trans. Antennas Propag. 59, 4436 (2011)
7. G. Minatti, S. Maci, P. De Vita, A. Freni, M. Sabbadini, A circularly-polarized isoflux antenna based on anisotropic metasurface, IEEE Trans. Antennas Propag. 60, 4998 (2012)

8. S. Pandi, C.A. Balanis, Curvature modeling in design of circumfrentially modulated cylindrical metasurface LWA, IEEE Antennas Wirel. Propag. Lett. 16, 1024 (2017)

9. A.A. Oliner, D.R. Jackson, Leaky-wave antennas, in: J.L. Volakis (Ed.), Antenna Engineering Handbook, 4th edn. (McGraw-Hill, New York, NY, 2007)

10. C. Walter, Traveling wave antennas (McGraw-Hill, New York, 1965)

11. M. Esquius-Morote, J.S. Gomez-Diaz, J. Perruisseau-Carrier, Sinusoidally modulated graphene leaky-wave antenna for electronic beamscanning at THZ, IEEE Trans. Terahertz Sci. Technol. 4, 116 (2014)

12. S.C. Pavone, E. Martini et al., Surface wave dispersion for a tunable grounded liquid crystal substrate without and with metasurface on top, IEEE Trans. Antennas Propag. 65, 3540 (2017)

Cite this article as: Doohyun Yang, Sangwook Nam, Electronically beamscannable sinusoidally modulated reactance surface antenna, EPJ Appl. Metamat. 6, 13 (2019) 